\title{
Análisis de la traqueotomía precoz y su impacto sobre la incidencia de neumonía, consumo de recursos y mortalidad en pacientes neurocríticos
}

\author{
F. Gandía-Martínez; I. Martínez-Gil; D. Andaluz-Ojeda; F. Bobillo de Lamo; L. Parra-Morais y F. Díez-Gutiérrez
}

Servicio de Medicina Intensiva. Hospital Clínico Universitario de Valladolid.

Resumen

Objetivos. Analizar el momento más adecuado para la realización de la traqueotomía en enfermos neurocríticos, comparando en una población seleccionada de pacientes las diferencias de morbimortalidad y consumo de recursos entre el grupo en que se realizó la traqueotomía precozmente ( $\leq 9$ días) y aquéllos en los que fue más tardía ( $>9$ días).

Material y métodos. Estudio prospectivo y observacional en una población de pacientes con diagnóstico de traumatismo craneoencefálico (TCE) o accidente cerebrovascular (ACVA) que precisaron traqueotomía durante su ingreso en UCI. Se compararon los datos en dos grupos de pacientes: a) traqueotomía precoz (TP) en los primeros 9 días; b) traqueotomía tardía (TT) a partir del $10^{\circ}$ día. Variables estudiadas: datos demográficos, gravedad al ingreso, procedencia, diagnóstico, duración de la intubación orotraqueal (IOT) y de la ventilación mecánica (VM), necesidades de sedación y de antibioterapia, frecuencia de neumonía asociada a ventilación mecánica (NAV), duración de la estancia y mortalidad. Se calculó el riesgo relativo de padecer neumonía y un modelo de regresión logística multivariante para determinar los factores asociados al desarrollo de neumonía. Significación estadística para una $\mathbf{p} \leq \mathbf{0 . 0 5}$.

Resultados. Se estudiaron 118 pacientes, $60 \%$ con TCE. La media de IOT previa a la traqueotomía (TRQ) fue de 12 días y la duración de la VM de 20 días. Se diagnosticaron 94 episodios de NAV en 81 pacientes $(68.6 \%)$. El grupo de TP muestra menor duración de la VM, de la sedación, de la antibioterapia y de la estancia en UCI, con menor incidencia de neumonía $(p<0.001)$. La precocidad de la TRQ no influyó en la duración de la estancia hospitalaria ( $p=0.844)$, ni en la mortalidad en UCI ( $(p=0.924)$, ni en la hospitalaria $(p=0.754)$. La media de edad fue menor en el grupo con TCE $(p<0.001)$,

Recibido: 31-07-09. Aceptado; 19-01-10 además la TRQ se realiza más tarde $(\mathrm{p}=0.026)$ y requieren más días de sedación $(\mathrm{p}=\mathbf{0 . 0 0 1})$ y de tratamiento antibiótico $(p=0.002)$. Los factores independientemente asociados con el desarrollo de neumonía fueron los días de IOT $(p=0.034$, OR 1.177) y los días de estancia en UCI ( $p=0.003$, OR 1.100). El riesgo relativo de padecer neumonía si la TRQ se realiza después de 9 días es 1.55 mayor (IC 95\%: 1.10-2.16). Número necesario de pacientes a tratar (NNT) para que la TRQ precoz evite un episodio de neumonía: 3.13. La presencia de NAV no se asocia con una mayor mortalidad en $\mathrm{UCI}(\mathrm{p}=\mathbf{0 . 5 5 8})$ ni hospitalaria $(\mathrm{p}=\mathbf{0 . 3 7 0})$.

Conclusiones. La traqueotomía precoz ( $\leq 9$ días) en los enfermos neurocríticos aporta ventajas apreciables, acortando los días de ventilación mecánica y de estancia en UCI, con menores requerimientos de sedación y de antibioterapia. Aunque la TRQ más tardía no se relaciona directamente con la mortalidad, se eleva considerablemente el riesgo de padecer neumonía, de manera especial en pacientes con TCE. Estas circunstancias clínicas deben valorarse individualmente en cada caso a fin de establecer el momento más adecuado para practicar la TRQ en los pacientes neurocríticos.

PALABRAS CLAVE: Traqueotomía. Traqueotomía precoz. Neurocríticos. Neumonía asociada a ventilación mecánica.

Analysis of early tracheostomy and its impact on development of pneumonia, use of resources and mortality in neurocritically ill patients

Abreviaturas. ACVA: accidente cerebrovascular agudo. APACHE II: acute physiology and chronic health evaluation. EPOC: enfermedad pulmonar obstructiva crónica. GCS: Glasgow coma score. IOT: intubación orotraqueal. NAV: neumonía asociada a ventilación mecánica. NNT: número necesario de pacientes a tratar. PIC: presión intracraneal. RR: riesgo relativo. TCE: traumantismo craneoencefálico. TP: traqueotomía precoz. TRQ: traqueotomía. TT: traqueotomía tardía. UCI: unidad de cuidados intensivos. VM: ventilación mecánica. 


\section{Summary}

Objective. We analyze the most suitable time to perform tracheostomy in neurocritically ill patients. We compare morbimortality and use of resources between those patients in which tracheostomy was done early ( $\leq 9$ days) and those in which it was perform later $(>9$ days), in a selected group of patients.

Material and methods. We made an observational prospective study involving a group of patients diagnosed as traumatic brain injury (TBI) or stroke, whose tracheostomy was performed during their stay at the Intensive Care Unit. We compared two groups: a) early tracheostomy (during first 9 days of ICU stay); b) late tracheostomy (made on 10th day or later). As variables, we studied: demographic data, severity of illness at admission, admittance department, diagnosis, length of intubation, length of mechanical ventilation (LMV), sedation and antibiotic treatment needs, ventilatorassociated pneumonia (VAP) events, ICU length of stay and mortality. We calculated relative risk of suffering from pneumonia and made a multivariate logistic regression to establish which factors were associated with an increased risk of developing pneumonia. Statistical signification $p<0.05$.

Results. We analyzed 118 patients, $60 \%$ with TBI. Mean length of intubation before tracheostomy was 12 days and mean LMV was 20 days. 94 VAP events were diagnosed in 81 patients $(68.6 \%)$. Early tracheostomy group showed lower length of mechanical ventilation and ICU stay, lower length of sedation and antibiotic treatment, and less pneumonia events $(p<0,001)$. The precocity of tracheostomy didn't have any influence either on hospital length of stay $(p=0.844)$, ICU mortality $(p=0.924)$ or in-hospital mortality $(p=0.754)$. At the TBI group mean age was lower $(p<0.001)$, tracheostomy was made later $(p=0.026)$, and patients needed a longer sedation $(p=0.001)$ and a longer antibiotic treatment $(p=0.002)$. Length of intubation $(p=0.034$, OR 1.177) and ICU length of stay $(\mathrm{p}=0.003$, OR 1.100) were factors independently associated with development of pneumonia. Relative risk of suffering from pneumonia when tracheostomy was made after 9 days of ICU stay was 1.55 (IC 95\%: 1.10-2.16). The number needed to treat (NNT) for early tracheostomy avoiding one pneumonia event was 3.13. VAP was not associated with a higher ICU ( $p=0.558)$ or in-hospital mortality $(p=0.370)$.

Conclusions. Early tracheostomy ( $\leq 9$ days) provides significant advantages in neurocritically ill patients: it shortens length of mechanical ventilation and ICU stay and decreases antibiotic and sedatives requirements. Although later tracheostomy is not directly related with mortality, it increases considerably the risk of suffering from pneumonia, particularly in patients with TBI. These clinical circumstances should be evaluated individually in each patient, so the best time to perform tracheostomy in neurocritically ill patients could be established.

KEY WORDS: Tracheostomy. Early tracheostomy. Neurocritically ill patients. Ventilator-associated pneumonia.

\section{Introducción}

La traqueotomía es un procedimiento frecuentemente realizado en los pacientes neurocríticos con la finalidad de facilitar el adecuado manejo respiratorio. Es una técnica indicada en los casos que precisan ventilación mecánica prolongada o cuando se requiere asegurar la permeabilidad de la vía aérea, estando asociada a una población de pacientes que se encuentra entre los que consumen una mayor cantidad de recursos sanitarios ${ }^{18,19}$. En comparación con la intubación translaríngea, la TRQ presenta potenciales ventajas: mejor tolerancia, mayor confort con menores requerimientos de analgesia y sedación, mayor seguridad de la vía aérea, reducción del espacio muerto y de la resistencia al flujo, mayor eficacia de las broncoaspiraciones, menores problemas fonatorios, acortamiento en el tiempo de "weaning" y de ventilación mecánica, capacidad de ingesta oral, menor frecuencia de neumonía nosocomial, y capacidad de transferir antes a los pacientes fuera de la unidad de cuidados intensivos (UCI), si bien no está definitivamente establecido que influya en la evolución clínica y la supervivencia de los pacientes en ventilación mecánica ${ }^{5,13,16,20,23,32}$. Aunque actualmente se considera que la realización de TRQ en la UCI es un procedimiento seguro, también puede conducir a serias complicaciones como la colonización bronquial o neumonía y la estenosis traqueal ${ }^{10,16,29}$.

La TRQ percutánea es una técnica de fácil aprendizaje y realización que actualmente se practica de manera habitual en la UCI a la cabecera del paciente. Tiene una baja incidencia de complicaciones y se ha convertido en un procedimiento rutinario en la práctica diaria para el manejo respiratorio de los pacientes críticos ${ }^{11,33}$. La disponibilidad de dicha técnica percutánea ha disminuido potencialmente el umbral para la realización de la TRQ en la UCI, aunque todavía no dispongamos de suficiente evidencia y exista controversia respecto a su impacto beneficioso sobre el paciente y su desenlace clínico $^{12,23}$. En la actualidad no existen datos ni recomendaciones definitivas que permitan determinar el tiempo óptimo para la realización de TRQ en diferentes grupos de pacientes con asistencia respiratoria, lo cual refleja la dificultad para predecir de manera precisa la duración de la VM. En estudios sobre diferentes poblaciones de pacientes médicos y quirúrgicos que precisan VM se han descrito resultados poco homogéneos, 
habiéndose publicado que la TRQ precoz facilita la liberación del respirador, disminuye la estancia en UCI y abarata los costes hospitalarios $6,7,9,15,24,26,30,31$, en algunos casos se describe adicionalmente una menor incidencia de neumonía que no se encuentra en otras series ${ }^{24,26,31}$, e incluso en determinados estudios se ha objetivado además una menor mortalidad hospitalaria ${ }^{9,24}$.

En pacientes neuroquirúrgicos con monitorización de la presión intracraneal (PIC) la realización de TRQ percutánea no causa complicaciones ni cambios clínicos significativos en la PIC, ni en la presión de perfusión cerebral, ni en el $\mathrm{GCS}^{27}$, estando considerado como un procedimiento útil y seguro. Comparada con la TRQ quirúrgica clásica, la técnica percutánea se ha relacionado con una menor estancia hospitalaria y menores costes económicos totales ${ }^{8}$. La TRQ electiva en pacientes neurocríticos seleccionados, con bajo GCS y neumonía nosocomial, podría permitir acortar la estancia en UCI y una más rápida retirada del respirador ${ }^{17,22,25}$.

Diferentes autores han encaminado sus esfuerzos a evaluar las posibles indicaciones y potenciales ventajas consecuentes a realizar precozmente la TRQ en pacientes con traumatismo craneoencefálico. Un número considerable de estudios en enfermos traumatológicos, con o sin TCE, encuentran que se acorta la duración de la VM y la estancia en UCI, aunque con conclusiones contradictorias en cuanto a la incidencia de neumonía y mortalidad ${ }^{1-4,14,34}$. Por el contrario, en otros trabajos clínicos llevados a cabo en pacientes similares no se demuestra ningún tipo de beneficio con la realización de TRQ precoz, puesto que no se acorta la duración de la estancia en UCI, ni se disminuyen los días de VM, ni se demuestra una menor incidencia de neumonía ni una disminución de la mortalidad ${ }^{5,35}$.

En este estudio hemos analizado en la población de pacientes neurocríticos más frecuente en UCI, como son los traumatismos craneoencefálicos y los accidentes cerebrovasculares, los datos de morbimortalidad y consumo de recursos en los enfermos traqueotomizados, comparando los resultados en los que se realizó la TRQ precozmente ( $\leq 9$ días) con los pacientes en que la TRQ fue más tardía ( $>9$ días). Como objetivo secundario hemos estudiado la posible existencia de características diferenciales entre el grupo de pacientes con TCE con relación a los ingresados por ACVA.

\section{Material y métodos}

Estudio prospectivo y observacional de datos clínicos seleccionados correspondientes a una población consecutiva de pacientes neurocríticos que a lo largo de cuatro años (2004-2007) fueron traqueotomizados en el Servicio de Medicina Intensiva de un hospital universitario. Todos los pacientes incluidos en el estudio eran adultos $(>16$ años), con diagnóstico de ingreso de TCE grave o accidente cerebrovascular agudo y que requirieron intubación orotraqueal y ventilación mecánica. Todos los enfermos habían superado la fase de reanimación y se encontraban estables, habiéndose retirado la monitorización de la presión intracraneal en los casos en que había sido necesaria. La decisión de realizar la TRQ fue establecida por el médico responsable, y motivada tanto por necesidad o expectativa de ventilación mecánica prolongada como por indicación de asegurar y proteger la vía aérea. La técnica utilizada preferentemente fue la percutánea sin control broncoscópico, practicando el procedimiento con sedoanalgesia y monitorización de la tensión arterial, saturación arterial de $\mathrm{O}_{2}$ y ECG. El facultativo responsable de practicar la TRQ indicó la técnica percutánea específica para su realización.

Se recogió información de las siguientes variables: Edad, sexo, gravedad al ingreso medida por el APACHE II (Acute Physiology and Chronic Health Evaluation), procedencia del paciente, diagnóstico al ingreso, días de IOT previos a la TRQ, días de VM, días de VM post TRQ, días con sedación, días con tratamiento antimicrobiano, incidencia de neumonía asociada a ventilación mecánica, días de estancia en UCI y en el hospital, mortalidad en UCI y mortalidad hospitalaria. Los pacientes fueron divididos en dos grupos en relación con el tiempo de IOT previo hasta la realización de la TRQ. A los que se les realizó la TRQ dentro de los primeros 9 días de IOT se les consideró en el grupo de TRQ precoz (TP), mientras que a los que se traqueotomizó a partir del $10^{\circ}$ día se les integró en el de TRQ tardía (TT). Adicionalmente comparamos las variables en los dos grupos de pacientes según el diagnóstico de ingreso: TCE versus ACVA.

El diagnóstico de NAV se estableció por medio de criterios clínicos y microbiológicos basados inicialmente en la sospecha clínica y en criterios clásicos, como son la presencia de nuevo infiltrado pulmonar persistente o progresivo visible en la radiografía de tórax y al menos dos de las siguientes condiciones: fiebre (dos o más determinaciones superior a $38^{\circ} \mathrm{C}$ ) o hipotermia, leucocitosis o leucopenia, esputo purulento o cambios en la naturaleza del esputo. La sospecha de NAV fue confirmada por la evolución clínica o por las técnicas microbiológicas cuantitativas habituales para las muestras bronquiales obtenidas por broncoaspirado o por cepillado bronquial ${ }^{29,36}$. En cada paciente se contabilizaron los episodios de neumonía padecidos a lo largo de toda su estancia en UCI, no siendo objeto de este trabajo el establecer comparaciones entre el número de infecciones previas y posteriores a la TRQ.

El análisis estadístico se realizó mediante el programa SPSS para Windows (SPSS 13.0, SPSS Inc., Chicago, IL). Para la comparación de variables continuas se utilizó el test t-Student, y para las variables categóricas el Chi2 de Pearson. Se calculó el riesgo relativo de padecer una neumonía 
Tabla 1

Datos demográficos y características clínicas de los pacientes neurocríticos estudiados

\begin{tabular}{|c|c|c|c|}
\hline \multicolumn{3}{|c|}{$\mathrm{N}^{o}$ de pacientes } & 118 \\
\hline \multicolumn{3}{|c|}{ Edad } & $52.3 \pm 19.2$ \\
\hline \multirow[t]{2}{*}{$\operatorname{Sexo}\left(n^{\circ}, \%\right)$ : } & \multicolumn{2}{|c|}{ Masculino } & $78(66.1 \%)$ \\
\hline & \multicolumn{2}{|c|}{ Femenino } & $40(33.9 \%)$ \\
\hline \multicolumn{3}{|l|}{ Apache II } & $19.2 \pm 5.8$ \\
\hline \multirow{3}{*}{\multicolumn{2}{|c|}{ Procedencia $\left(\mathrm{n}^{\circ}, \%\right)$ : }} & Urgencias & $59(50 \%)$ \\
\hline & & Traslado & $41(35.7 \%)$ \\
\hline & & Hospitalización & $18(14.3 \%)$ \\
\hline \multirow{2}{*}{\multicolumn{2}{|c|}{ Diagnóstico $\left(\mathrm{n}^{\mathrm{o}}, \%\right)$ : }} & TCE & $71(60.2 \%)$ \\
\hline & & ACVA & $47(39.8 \%)$ \\
\hline \multicolumn{3}{|c|}{ IOT hasta la TRQ (d) } & $12.1 \pm 8.4$ \\
\hline \multicolumn{3}{|c|}{ Ventilación mecánica (d) } & $20.2 \pm 13.9$ \\
\hline \multicolumn{3}{|c|}{ VM post TRQ (d) } & $8.8 \pm 12.8$ \\
\hline \multicolumn{3}{|c|}{ Sedación (d) } & $13.1 \pm 10.7$ \\
\hline \multicolumn{3}{|c|}{ Antibióticos (d) } & $21.0 \pm 14.5$ \\
\hline \multicolumn{3}{|c|}{ Pacientes con neumonía $\left(\mathrm{n}^{\circ}, \%\right)$} & $81(68.6 \%)$ \\
\hline \multicolumn{3}{|c|}{ Estancia en UCI (d) } & $26.5 \pm 14.9$ \\
\hline \multicolumn{3}{|c|}{ Estancia hospitalaria (d) } & $49.1 \pm 28.7$ \\
\hline \multicolumn{3}{|c|}{ Mortalidad UCI $\left(\mathrm{n}^{\mathrm{o}}, \%\right)$} & $13(11 \%)$ \\
\hline \multicolumn{3}{|c|}{ Mortalidad hospitalaria $\left(\mathrm{n}^{\mathrm{o}}, \%\right)$} & $23(19.5 \%)$ \\
\hline
\end{tabular}

TCE: traumatismo craneoencefálico. ACVA: accidente cerebrovascular agudo. VM: ventilación mecánica. IOT: intubación orotraqueal. TRQ: Traqueotomía. UCI: Unidad de Cuidados Intensivos. Cuando corresponde los valores se expresan como media \pm desviación estándar de la media. (d): días.

(RR) y el número necesario de pacientes a tratar para evitarla (NNT). El análisis de regresión logística multivariante para determinar los factores independientemente asociados con el desarrollo de NAV se realizó utilizando un modelo por pasos hacia atrás ajustado por edad, sexo y Apache II, incluyendo las variables asociadas con un valor de $\mathrm{p} \leq 0.2$ en el análisis univariante, umbral que determina la admisión en el modelo. Se incluyeron las siguientes variables: edad, sexo, Apache II, diagnóstico, días de sedación, días de intubación orotraqueal, días de ventilación mecánica y estancia en UCI. Se consideró significación estadística una $\mathrm{p} \leq 0.5$.

\section{Resultados}

En la Tabla 1 se muestran los datos demográficos, las características clínicas y la morbimortalidad global de los 118 pacientes, $71(60 \%)$ diagnosticados de TCE y 47 (40\%) de ACVA, con intubación translaríngea y TRQ prac- ticada en la UCI que fueron incluidos en el estudio. Salvo contraindicación la técnica de elección para la realización de TRQ fue la percutánea, practicándose dicha técnica en 106 pacientes (90\%), mientras en el resto se realizó TRQ quirúrgica. El grupo de 47 pacientes con el diagnóstico de accidente cerebrovascular tenían los siguientes diagnósticos etiológicos: hemorragia cerebral 21 (46.8\%), $\mathrm{ACV}$ isquémico 17 (36.2\%) y hemorragia subaracnoidea $9(17 \%)$. Predominan los varones $(66.1 \%)$ y el diagnóstico de TCE grave (60\%), ingresando el 50\% de los pacientes estudiados a través de urgencias con un elevado APACHE II (19.2 \pm 5.8$)$. La media de intubación translaríngea fue de $12.1 \pm 8.4$ días y la de ventilación mecánica de $20.2 \pm 13.9$ días. A lo largo de la estancia se diagnosticaron un total de 94 episodios de NAV, afectándose el $68.6 \%$ (81 pacientes). En 69 enfermos se detectó un episodio de NAV, 2 episodios en 11 pacientes y en uno se produjeron 3 episodios de neumonía durante su estancia en UCI.

La comparación de variables entre el grupo de TRQ precoz y TRQ tardía se muestra en la Tabla 2. No se encuentran diferencias en la edad $(\mathrm{p}=0.901)$, sexo $(\mathrm{p}=0.115)$, APACHE II al ingreso $(\mathrm{p}=0.899)$ ni en el número de días de VM una vez practicada la TRQ ( $\mathrm{p}=0.243$ ). En el grupo de TT existe una proporción mayor de pacientes con TCE que con ACVA (69.2\% versus $30.8 \%, \mathrm{p}=0.026)$. Hemos encontrado diferencias significativas $(\mathrm{p}<0.001)$ en la incidencia de neumonía, en los días de intubación orotraqueal y de ventilación mecánica, en la duración de la sedación y de los tratamientos con antimicrobianos, así como en la estancia en UCI, siendo los valores significativamente superiores cuando la TRQ se realiza a partir del $10^{\circ}$ día. Sin embargo, la precocidad de la TRQ no influyó en los días de ventilación mecánica post TRQ ( $\mathrm{p}=0.243)$, ni en la duración de la estancia hospitalaria $(\mathrm{p}=0.844)$, ni en la mortalidad en UCI ( $\mathrm{p}=0.924) \mathrm{u}$ hospitalaria (0.754). En el grupo de TP se produjeron 29 episodios de neumonía en 27 pacientes, mientras que en el de TT se diagnosticaron 65 episodios entre los 54 pacientes que sufrieron NAV $(\mathrm{p}=0.001)$.

Comparados con los pacientes diagnosticados de ACVA (Tabla 3), los enfermos con TCE tienen menor edad $(\mathrm{p}<0.001)$, la TRQ se realiza más tarde $(\mathrm{p}=0.026)$, y requieren más días de sedación $(\mathrm{p}=0.001)$ y de tratamiento antibiótico ( $\mathrm{p}=0.002)$. Igualmente, la incidencia de neumonía y la estancia en UCI es mayor en el TCE, pero sin llegar a la significación estadística $(\mathrm{p}=0.084$ y $\mathrm{p}=0.059$, respectivamente). Encontramos una mayor mortalidad hospitalaria en el grupo de ACVA, sin llegar tampoco a ser significativa la diferencia $(\mathrm{p}=0.068)$. La Figura 1 compara en cada grupo diagnóstico (TCE y ACVA) el porcentaje de pacientes a los que se realizó TP en relación con TT. A los pacientes con ACVA se les realiza la TRQ más precozmente que al grupo con TCE ( $p=0.026)$. Igualmente, en la Figura 2 se muestra la misma comparación en relación con la duración 


\section{Tabla 2}

Comparación entre TRQ precoz y TRQ tardía en relación con el tiempo de intubación orotraqueal hasta la realización de TRQ

\begin{tabular}{|c|c|c|c|c|}
\hline & & $\begin{array}{l}\text { TRQ } \leq 9 \text { días } \\
(\mathrm{n}=53)\end{array}$ & $\begin{array}{l}\text { TRQ }>9 \text { días } \\
\quad(n=65)\end{array}$ & $\mathrm{p}$ \\
\hline \multicolumn{2}{|l|}{ Edad } & $52.1 \pm 19.6$ & $52.5 \pm 19.0$ & 0.901 \\
\hline \multirow{2}{*}{ Sexo $\left(n^{0}, \%\right)$ : } & Masculino & $31(58.5 \%)$ & $47(72.3 \%)$ & 0.115 \\
\hline & Femenino & $22(41.5 \%)$ & $18(27.7 \%)$ & \\
\hline \multirow[t]{2}{*}{ Diagnóstico $\left(\mathrm{n}^{\mathrm{o}}, \%\right)$ : } & TCE & $26(49.1 \%)$ & $45(69.2 \%)$ & 0.026 \\
\hline & ACVA & $27(50.9 \%)$ & $20(30.8 \%)$ & \\
\hline \multicolumn{2}{|l|}{ Apache II } & $19.2 \pm 5.6$ & $19.1 \pm 6.0$ & 0.899 \\
\hline \multicolumn{2}{|l|}{ GCS } & $7.1 \pm 3.2$ & $7.4 \pm 3.6$ & 0.784 \\
\hline \multicolumn{2}{|c|}{ IOT hasta la TRQ (d) } & $7.1 \pm 1.5$ & $15.6 \pm 7.7$ & $<0.001$ \\
\hline \multicolumn{2}{|c|}{ Ventilación mecánica (d) } & $14.1 \pm 8.5$ & $25.2 \pm 15.1$ & $<0.001$ \\
\hline \multicolumn{2}{|c|}{ VM post TRQ (d) } & $6.9 \pm 9.2$ & $9.5 \pm 12.9$ & 0.243 \\
\hline \multicolumn{2}{|l|}{ Sedación (d) } & $8.1 \pm 5.3$ & $17.2 \pm 12.2$ & $<0.001$ \\
\hline \multicolumn{2}{|c|}{ Antimicrobianos (d) } & $15.4 \pm 11.6$ & $25.4 \pm 14.6$ & $<0.001$ \\
\hline \multicolumn{2}{|c|}{ Pacientes con NAV $\left(\mathrm{n}^{\circ}, \%\right)$} & $27(50.9 \%)$ & $54(83.1 \%)$ & $<0.001$ \\
\hline \multicolumn{2}{|c|}{ Estancia en UCI (d) } & $20.7 \pm 10.2$ & $31.2 \pm 16.3$ & $<0.001$ \\
\hline \multicolumn{2}{|c|}{ Estancia hospitalaria (d) } & $48.6 \pm 30.6$ & $49.62 \pm 27.9$ & 0.844 \\
\hline \multicolumn{2}{|c|}{ Mortalidad UCI $\left(\mathrm{n}^{\circ}, \%\right)$} & $6(11.3 \%)$ & $7(10.8 \%)$ & 0.924 \\
\hline \multicolumn{2}{|c|}{ Mortalidad hospitalaria (no,$\%)$} & $11(20.8 \%)$ & $12(18.5 \%)$ & 0.754 \\
\hline
\end{tabular}

TCE: traumatismo craneoencefálico. ACVA: accidente cerebrovascular agudo. VM: ventilación mecánica. IOT: intubación orotraqueal. TRQ: Traqueotomía. NAV: neumonía asociada a ventilación mecánica. GCS: Glasgow coma score. Cuando corresponde los valores se expresan como media \pm desviación estándar de la media. (d): días.

Tabla 3

Comparación entre pacientes con TCE y ACVA

\begin{tabular}{|c|c|c|c|}
\hline & $\begin{array}{l}\text { TCE } \\
(\mathrm{n}=71)\end{array}$ & $\begin{array}{l}\text { ACVA } \\
(\mathrm{n}=47)\end{array}$ & $\mathrm{p}$ \\
\hline Edad & $47.3 \pm 21.4$ & $59.9 \pm 12.2$ & $<0.001$ \\
\hline Sexo masculino $\left(\mathrm{n}^{\circ}, \%\right)$ & $49(69 \%)$ & $29(61.7 \%)$ & 0.411 \\
\hline Apache II & $18.9 \pm 5.3$ & $19.4 \pm 6.5$ & 0.676 \\
\hline IOT hasta la TRQ (d) & $12.2 \pm 6.9$ & $11.8 \pm 10.3$ & 0.835 \\
\hline Ventilación mecánica (d) & $21.4 \pm 12.1$ & $18.5 \pm 16.2$ & 0.287 \\
\hline VM post TRQ (d) & $9.2 \pm 11.1$ & $8.2 \pm 15.4$ & 0.691 \\
\hline $\mathrm{TRQ} \leq 9$ días $\left(\mathrm{n}^{\mathrm{o}}, \%\right)$ & $26(36.6 \%)$ & $27(57.4 \%)$ & 0.026 \\
\hline Sedación (días) & $15.7 \pm 11.4$ & $9.2 \pm 8.3$ & 0.001 \\
\hline Antimicrobianos (d) & $24.4 \pm 14.4$ & $15.9 \pm 13.1$ & 0.002 \\
\hline Pacientes con NAV $\left(\mathrm{n}^{\circ}, \%\right)$ & $53(74.6 \%)$ & $28(59.6 \%)$ & 0.084 \\
\hline Estancia en UCI (d) & $28.6 \pm 13.6$ & $23.3 \pm 16.2$ & 0.059 \\
\hline Estancia hospitalaria (d) & $47.1 \pm 24.2$ & $52.2 \pm 34.5$ & 0.347 \\
\hline Mortalidad UCI $\left(\mathrm{n}^{\circ}, \%\right)$ & $6(8.5 \%)$ & $7(14.9 \%)$ & 0.274 \\
\hline Mortalidad hospitalaria $\left(n^{\circ}, \%\right)$ & $10(14.1 \%)$ & $13(27.7 \%)$ & 0.068 \\
\hline
\end{tabular}

TCE: traumatismo craneoencefálico. ACVA: accidente cerebrovascular agudo. VM: ventilación mecánica. IOT: intubación orotraqueal. TRQ: traqueotomía. NAV: neumonía asociada a ventilación mecánica. Cuando corresponde los valores se expresan como media \pm desviación estándar de la media. (d): días. 


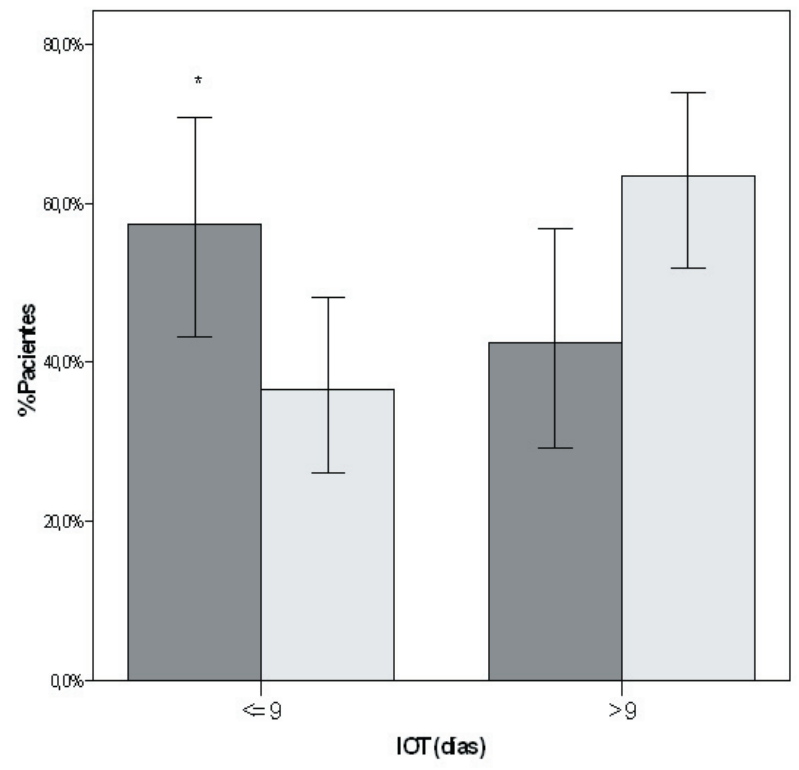

Figura 1. Comparación del porcentaje de pacientes con ACVA versus TCE en ambos grupos de traqueotomía precoz ( $\leq 9$ dias) y tardía (>9 días). Barras oscuras: accidentes cerebrovasculares (ACVA). Barras claras: traumatismos craneoencefálicos (TCE). IOT: intubación orotraqueal. Grupo de traqueotomía precoz: ACVA vs TCE, * $p=0.026$ (IC 95\%)

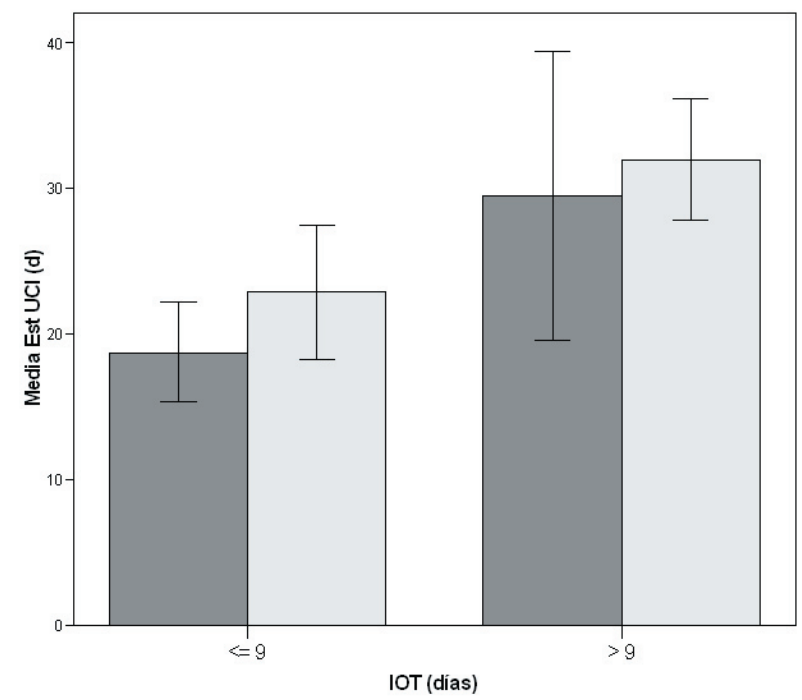

Figura 3. Comparación de la estancia en UCI entre TCE y ACVA en ambos grupos de traqueotomía precoz ( $\leq 9$ días) y tardía (>9 días). Barras oscuras: ACVA. Barras claras: TCE. UCI: unidad de cuidados intensivos. IOT: intubación orotraqueal. Sin diferencia significativa entre TCE y ACVA en ambos grupos.

de la ventilación mecánica y en la Figura 3 las diferencias

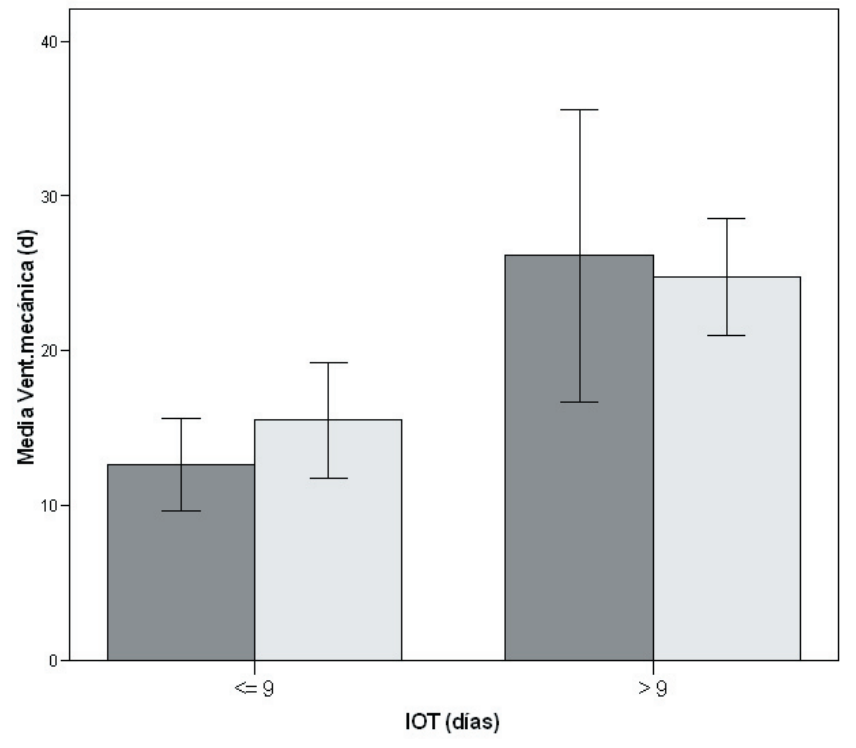

Figura 2. Comparación de la duración de la ventilación mecánica entre TCE y ACVA en ambos grupos de traqueotomía precoz ( $\leq 9$ dias) y tardía ( $>9$ días). Barras oscuras: ACVA. Barras claras: TCE. TRQ: traqueotomía. IOT intubación orotraqueal. TCE vs ACVA: sin diferencias significativas en la duración de la ventilación mecánica tanto en el grupo de TRQ precoz como en TRQ tardía.

con respecto a la estancia en UCI. En ambos casos no se encuentran diferencias significativas entre el grupo con TCE y el grupo con ACVA.

En la Tabla 4 se describen los resultados de la comparación entre los enfermos que desarrollaron NAV con los que no la padecieron. La complicación neumónica aparece con mayor frecuencia en el sexo masculino $(p=0.022)$, en aquellos pacientes en que la TRQ se realiza más tarde $(\mathrm{p}<0.001)$, que están más tiempo en $\mathrm{VM}(\mathrm{p}<0.001)$ y que por tanto necesitan más sedación, tratamiento antibiótico más prolongado $(\mathrm{p}<0.001)$ y una más larga estancia en UCI $(p<0.001)$ y hospitalaria $(p=0.017)$. Sin embargo, el desarrollo de neumonía no influye en la mortalidad intra UCI $(\mathrm{p}=0.558)$ ni en la hospitalaria $(\mathrm{p}=0.370)$. La mayor incidencia de NAV en pacientes con TCE comparada con los enfermos de ACVA no llega a ser significativa $(\mathrm{p}=0.084)$. En la Figura 4 mostramos la comparación en la incidencia de NAV entre los pacientes con TRQ precoz y en los que se realiza TRQ tardía, objetivándose mayor frecuencia de infección neumónica en el grupo en que la traqueotomía se practica tardíamente $(\mathrm{p}<0.001)$.

Los resultados del modelo de regresión logística multivariante y del riesgo relativo se muestran en la Tabla 5. En el análisis multivariante los factores independientemente asociados con el desarrollo de NAV fueron los días de IOT ( $\mathrm{p}=0.034$, OR 1.177 , IC 95\%: 1.013-1.369) y 
Tabla 4

Comparación entre pacientes con y sin neumonía asociada a ventilación mecánica

\begin{tabular}{|c|c|c|c|c|}
\hline & & $\begin{array}{l}\text { No NAV } \\
(\mathrm{n}=37)\end{array}$ & $\begin{array}{l}\text { NAV } \\
(\mathrm{n}=81)\end{array}$ & $\mathrm{p}$ \\
\hline \multicolumn{2}{|l|}{ Edad } & $55.5 \pm 20.3$ & $50.8 \pm 18.6$ & 0.221 \\
\hline \multirow[t]{2}{*}{$\operatorname{Sexo}\left(n^{0}, \%\right)$ : } & masculino & $19(51.4 \%)$ & $59(72.8 \%)$ & 0.022 \\
\hline & femenino & $18(48.6 \%)$ & $22(27.2 \%)$ & \\
\hline \multirow[t]{2}{*}{ Diagnóstico $\left(\mathrm{n}^{\mathrm{o}}, \%\right)$ : } & TCE & $18(48.6 \%)$ & $53(65.4 \%)$ & 0.084 \\
\hline & ACVA & $19(51.4 \%)$ & $28(34.6 \%)$ & \\
\hline \multicolumn{2}{|l|}{ Apache II } & $19.4 \pm 5.3$ & $19.0 \pm 6.1$ & 0.742 \\
\hline \multicolumn{2}{|c|}{$\mathrm{IOT}(\mathrm{d})$} & $8.4 \pm 3.5$ & $13.4 \pm 7.9$ & $<0.001$ \\
\hline \multicolumn{2}{|c|}{ Ventilación mecánica (d) } & $13.2 \pm 5.8$ & $23.4 \pm 15.0$ & $<0.001$ \\
\hline \multicolumn{2}{|c|}{ VM post TRQ (d) } & $4.7 \pm 5.1$ & $10.0 \pm 13.1$ & 0.020 \\
\hline \multicolumn{2}{|l|}{ TRQ $>9$ días $\left(\mathrm{n}^{\circ}, \%\right)$} & $11(29.7 \%)$ & $54(66.7 \%)$ & $<0.001$ \\
\hline \multicolumn{2}{|l|}{ Sedación (días) } & $7.6 \pm 5.8$ & $15.6 \pm 11.5$ & $<0.001$ \\
\hline \multicolumn{2}{|l|}{ Antimicrobianos (d) } & $9.7 \pm 8$ & $26.1 \pm 13.5$ & $<0.001$ \\
\hline \multicolumn{2}{|c|}{ Estancia en UCI (d) } & $18.0 \pm 6.4$ & $30.4 \pm 15.9$ & $<0.001$ \\
\hline \multicolumn{2}{|c|}{ Estancia hospitalaria (d) } & $39.8 \pm 21.2$ & $53.3 \pm 30.7$ & 0.017 \\
\hline \multicolumn{2}{|c|}{ Mortalidad UCI $\left(\mathrm{n}^{\circ}, \%\right)$} & $5(13.5 \%)$ & $8(9.9 \%)$ & 0.558 \\
\hline \multicolumn{2}{|c|}{ Mortalidad hospitalaria (no $\%$ ) } & $9(24.3 \%)$ & $14(17.3 \%)$ & 0.370 \\
\hline
\end{tabular}

NAV: neumonía asociada a ventilación mecánica. TCE: traumatismo craneoencefálico. ACVA: accidente cerebrovascular agudo. VM: ventilación mecánica. IOT: intubación orotraqueal. TRQ: traqueotomía. Cuando corresponde los valores se expresan como media \pm desviación estándar de la media. (d): días

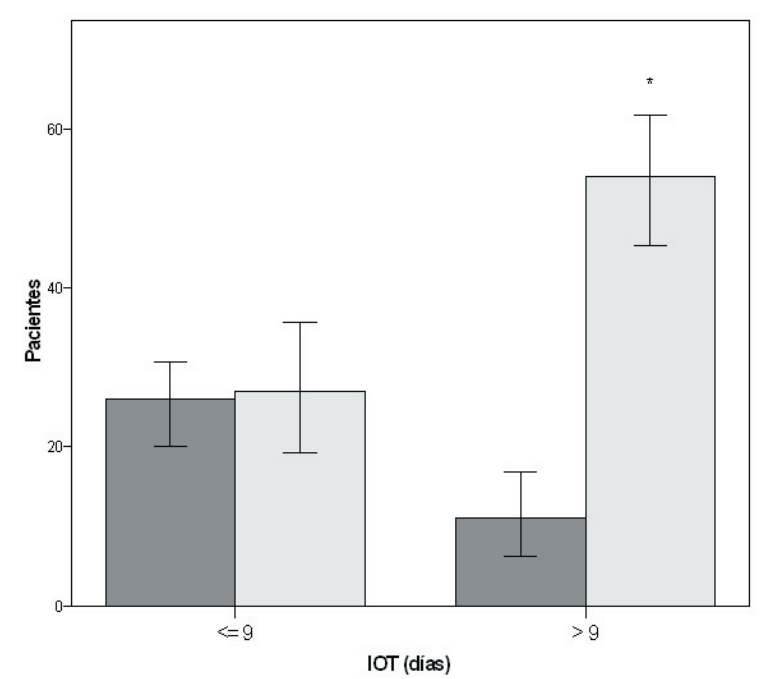

Figura 4. Comparación de pacientes con NAV entre el grupo de precoz ( $\leq 9$ días) y Tardía (> 9 días). Barras oscuras. Pacientes sin NAV. Barras claras: pacientes con $N A V . *$ Comparación del número de pacientes con NAV en $T R Q \leq 9$ días y TRQ $>9$ días: $p<0.001$. NAV: neumonía asociada a ventilación mecánica.

los días de estancia en UCI ( $\mathrm{p}=0.003$, OR 1.100 , IC 95\%:
1.032-1.177). El riesgo relativo de padecer NAV si la TRQ se realiza después de 9 días de intubación orotraqueal es de 1.55 (IC 95\%: 1.10-2.16). El número necesario de pacientes a tratar (NNT) es de 3.13, es decir, por cada 3 pacientes en que se realiza la TRQ en los primeros 9 días se evita una neumonía.

\section{Discusión}

En el entorno clínico de los cuidados críticos la traqueotomía es considerada todavía y desde mucho tiempo atrás como un procedimiento a realizar como último recurso en pacientes que dependen de un respirador. La disponibilidad de la técnica percutánea a la cabecera del paciente, su fácil aprendizaje y la baja incidencia de complicaciones ha provocado un considerable incremento en su frecuencia de realización para facilitar el manejo respiratorio prolongado de los pacientes neurocríticos. En casos de hemorragia cerebral espontánea el 10\% de los pacientes requieren TRQ durante el curso de su enfermedad, siendo factores de riesgo la presencia de EPOC, el volumen del hematoma, su localización en ganglios basales y el desarrollo de hidrocefalia ${ }^{21}$. En los enfermos con lesiones neurológicas infratentoriales de diferente etio- 


\section{Tabla 5}

Análisis multivariante y riesgo relativo de padecer neumonía en los pacientes neurocríticos motivo de estudio

Análisis multivariante

\begin{tabular}{ccc} 
OR & IC95\% & $\mathrm{p}$ \\
\hline 1.177 & $1.013-1.369$ & 0.034 \\
1.100 & $1.032-1.177$ & 0.003
\end{tabular}

Riesgo relativo

Riesgo relativo de padecer NAV si TRQ $>9$ días:

Número necesario de pacientes a tratar:
$\mathrm{RR}=1.55$

$\mathrm{NNT}=3.13$
(IC 95\%: 1.10-2.16).

IOT: intubación orotraqueal. NAV: neumonía asociada a ventilación mecánica. RR: riesgo relativo. NNT: Número necesario de pacientes a los que realizar traqueotomía precoz para evitar un episodio de neumonía.

logía (traumáticas, vasculares y tumorales) la frecuencia de TRQ es del $33 \%$, recomendándose una política agresiva hacia la realización de TRQ al $8^{\circ}$ día basándose en el alto porcentaje de reintubaciones y traqueotomías en este tipo de lesiones ${ }^{28}$. En la actualidad, y aunque un considerable número de enfermos podría en buena lógica beneficiarse precozmente de las potenciales ventajas de la TRQ, los criterios de selección en los diferentes grupos de pacientes están sometidos todavía a investigación y controversia en relación al tiempo idóneo para practicar una TRQ. Hasta donde conocemos este estudio es el primero que evalúa las posibles ventajas de realizar la TRQ precozmente (en los primeros 9 días de IOT) en un grupo de pacientes que representan la población más frecuente y representativa de los enfermos neurocríticos ingresados en UCI que precisan intubación translaríngea y ventilación mecánica prolongadas, como son los pacientes con TCE y con ACVA.

En consonancia con los resultados de otros autores $^{1,4,25,27}$, en nuestra serie los pacientes neurocríticos traqueotomizados son preferentemente varones (66\%), con un elevado nivel medio de gravedad al ingreso (Apache II: 19). La mortalidad hospitalaria fue del $19.5 \%$, inferior a la esperable y a la descrita globalmente para todos los pacientes a los que se realiza TRQ en $\mathrm{UCI}^{9,12,24}$, lo cual podría estar relacionado con la elevada proporción de pacientes más jóvenes con TCE $(60 \%$ del total, con mortalidad hospitalaria del $14.1 \%$ ) y con la ausencia de enfermos de causa primariamente respiratoria o séptica, más susceptibles inicialmente de desembocar en fracaso multiorgánico. La duración media de la IOT previa a la realización de TRQ fue de 12 días, y la de la ventilación mecánica fue de 20 días, objetivándose largas estancias tanto en la UCI (media 26 días) como hospitalaria (media 49 días). Estas características clínicas pueden considerarse en la línea con las previamente descritas por otros autores en otros tipos de pacientes críticos: polivalentes, neurocríticos, médicos, quirúrgicos, politraumatizados y $\mathrm{TCE}^{1-4,6-9,12,13,17,22,26}$.

En pacientes traumatológicos con o sin TCE existen resultados dispares en relación con las posibles ventajas de realizar precozmente la TRQ. Gurkin et al. encontraron que los casos de traumatismo craneoencefálico con GCS $\leq 8$ requieren frecuentemente la realización de TRQ, y que practicarla en los primeros 7 días disminuye la morbilidad y la duración de la estancia hospitalaria ${ }^{17}$. Otros autores coinciden en la necesidad de la realización precoz de TRQ (tan pronto como pueda ser razonablemente tolerado el procedimiento) en pacientes con TCE que presentan GCS $<7$ y SAPS $>15$ al $4^{\circ}$ día de ingreso ${ }^{25}$. Tanto en politraumatismos como en casos de TCE aislado la TRQ precoz acortaría la duración de la VM y la estancia en UCI, en algunas series con disminución en la incidencia de neumonía, sin repercusión en el desenlace clínico y con disminución en el consumo de recursos sanitarios ${ }^{1-4,14,30,34}$. Rumbak. et al describen incluso una disminución en la mortalidad en pacientes en VM que se prevee precisen IOT $>14$ días si la TRQ se realiza dentro de las primeras 48 horas $^{31}$. Por el contrario, Barquist et al concluyen que, en pacientes traumatizados dependientes del respirador la estrategia de practicar la TRQ antes de los 8 días no reduce los días de VM, ni la duración de la estancia en UCI, ni la frecuencia de neumonía cuando se compara con el grupo de traqueotomizados a partir del día $28^{5}$. En un estudio multicéntrico, prospectivo y randomizado, realizado tanto en pacientes con TCE, como en politraumatizados, como en enfermos no traumatológicos, Sugerman et al no encuentran diferencias significativas en la duración de la estancia en UCI, ni 
en la frecuencia de neumonía, ni en la mortalidad, ni en las complicaciones estenóticas laríngeas post intubación translaríngea comparando ambos grupos de TRQ precoz (3-5 días) y TRQ tardía (10-14 días) con los pacientes que seguían con intubación translaríngea ${ }^{35}$.

En la población de pacientes neurocríticos motivo de éste estudio, comparando el grupo en que se practica la TRQ precoz ( $\leq 9$ días) con el de TRQ tardía ( $>9$ días), hemos objetivado que en los primeros se acorta el tiempo de ventilación mecánica con menores necesidades de sedación, presentan una inferior incidencia de NAV con menores requerimientos de tratamientos antibióticos, y se disminuye significativamente la estancia en la UCI. Por el contrario, y para un similar nivel de gravedad al ingreso (Apache II 19 en ambos grupos), la TRQ precoz no influye significativamente en la estancia hospitalaria ni en la mortalidad. Como ya se ha expuesto, los enfermos de nuestra serie no son estrictamente comparables a los anteriormente descritos por otros autores, pero nuestros resultados se alinearían en mayor o menor medida con los publicados sobre el impacto beneficioso de la TRQ precoz en la evolución clínica y resultados en diferentes grupos de pacientes neurocríticos ${ }^{1,4,17,22,26}$.

En este estudio, siendo nuestros pacientes previamente no infecciosos, a lo largo de su ingreso en UCI el $68 \%$ desarrollaron al menos un episodio de NAV. Esta es una frecuente complicación de los pacientes sometidos a ventilación mecánica, con un riesgo mayor en politraumatismos (45\%) que en aquellos casos con TCE aislado, asociándose su desarrollo a un mayor grado de disfunción orgánica noneurológica ${ }^{36}$. Se ha descrito una amplia variabilidad en la incidencia de NAV en pacientes traqueotomizados. Rello et al en una población polivalente, de los cuales el $80 \%$ recibían tratamiento antibiótico previo a la TRQ y el $20 \%$ tenía tratamiento corticoideo, encuentra una incidencia de NAV del $18 \%$. Boynton et al en pacientes similares describe una frecuencia de NAV del $27 \%^{6}$, Chia-Lin et al del $66.9 \%{ }^{24}$ y Rumbak et al del $20 \%{ }^{31}$. En TCE se ha descrito una frecuencia de NAV entre el $45 \%$ y el $61 \%^{1,4}$. En un reciente metaanálisis Griffiths et al concluyen que aunque la TRQ precoz acorta el tiempo de ventilación mecánica y los días de estancia en UCI, no influye en la frecuencia de padecer neumonía hospitalaria ${ }^{15}$. La inhomogeneidad de las poblaciones de pacientes estudiados, las diferencias en los criterios utilizados para el diagnóstico de neumonía, así como los límites de la precisión diagnóstica clínica y microbiológica de NAV podrían explicar esta disparidad de resultados. En cualquier caso, nosotros hemos encontrado una elevada incidencia de NAV en los pacientes neurocríticos traqueotomizados, especialmente alta en los pacientes con TCE.

Comparados con el grupo de TCE, a una mayor proporción de pacientes con ACVA se les practicó la TRQ antes de los 9 días, motivado probablemente porque su grave situación neurológica residual hacía preveer de manera más evidente la prolongada necesidad de asistencia respiratoria y/o aislamiento de la vía aérea. Por otra parte, siendo éste un estudio observacional que analiza la realización de TRQ en pacientes neurocríticos una vez retirada la monitorización de la PIC cuando ésta ha sido necesaria, un posible sesgo en nuestros resultados podría derivarse de que en el grupo de TRQ tardía hubiera una mayor presencia de casos en que la PIC fue más elevada o de manejo más difícil y, por tanto, de peor evolución, lo que retrasaría la realización de TRQ. Razonablemente los pacientes con TCE podrían haber requerido un tiempo más prolongado de monitorización de la PIC, lo cual podría contribuir a una mayor proporción de TRQ tardía en este grupo que en el de ACVA. En todo caso, y como se ha comentado anteriormente, la realización de TRQ percutánea en pacientes con monitorización de la PIC está considerado un procedimiento seguro exento de complicaciones y de cambios significativos en la presión intracraneal ${ }^{27}$.

Aunque los pacientes con TCE requirieron una sedación y tratamiento antibiótico más prolongados no hubo diferencias entre ambos grupos en la duración total de la VM, y sin embargo la mayor frecuencia de NAV en el TCE roza la significación estadística. Es decir, para niveles similares de Apache II y número de días de VM los pacientes con TCE tuvieron una mayor frecuencia (en el límite de la significación estadística) de NAV que los enfermos con ACVA. No podemos contrastar estos hallazgos con otros trabajos que hayan estudiado una población similar de pacientes, y no ha sido objetivo nuestro el analizar los traumatismos asociados a nuestros pacientes con TCE, pero de acuerdo con los hallazgos de Zygun et $a^{36}$, la presencia de otras lesiones traumáticas asociadas al TCE, especialmente el trauma torácico, y las frecuentes complicaciones orgánicas no neurológicas secundarias al propio traumatismo y a las medidas habituales de reanimación podrían explicar esta mayor incidencia de NAV en los enfermos con TCE. Las diferentes características clínicas entre ambos grupos de pacientes podrían explicar, también en el límite de la significación estadística, la mayor estancia en UCI de los enfermos con TCE (mayor necesidad de sedación e incidencia de NAV) y la mayor mortalidad hospitalaria de los enfermos vasculares (mayor edad, más comorbilidades, lesiones neurológicas residuales post ictus).

La NAV no se ha mostrado como un factor de riesgo directamente relacionado con la mortalidad, sin embargo su frecuencia es mayor en la TRQ tardía, con mayores requerimientos en el tiempo de VM, mayor consumo de recursos sanitarios y una estancia más prolongada en UCI y en el hospital. Tanto la duración de la IOT previa a la TRQ como los días de estancia en UCI son factores independientemente asociados con el desarrollo de neumonía, factores 
susceptibles de ser modificados y mejorados mediante la realización precoz de la TRQ. El riesgo relativo de padecer NAV si la TRQ se realiza después de 9 días de intubación orotraqueal es 1.55 veces mayor, y por cada 3 pacientes en que se realiza la TRQ en los primeros 9 días se evita un episodio de neumonía. Aún así, la NAV no fue un factor de riesgo independientemente asociado con la mortalidad.

En resumen, en la población de pacientes neurocríticos ingresados en UCI que más frecuentemente precisan TRQ (TCE y ACVA), la realización de dicha técnica en los primeros 9 días de IOT acorta el tiempo de VM y la consiguiente necesidad de sedación, disminuye la frecuencia de neumonía acortando los tratamientos antimicrobianos, y disminuye la estancia en UCI sin modificar la duración de la estancia hospitalaria ni la mortalidad en UCI u hospitalaria. Al grupo de pacientes con TCE se les practica la TRQ más tarde que a los neurocríticos vasculares, por lo que la realización de la TRQ más precozmente podría disminuir la elevada incidencia de neumonía asociada a ventilación mecánica que muestra este subgrupo de pacientes traumatológicos. Finalmente, aunque todavía no dispongamos de suficiente evidencia como para indicar en todos los casos el tiempo oportuno para la práctica de la TRQ, con los conocimientos actuales resulta anacrónico y carente de evidencia esperar a que el calendario cumpla los 14-21 días de IOT para realizar una TRQ en los pacientes neurocríticos, en los que la TRQ precoz ha mostrado evidentes ventajas. En nuestra opinión, la decisión clínica de practicar dicha técnica en el enfermo neurocrítico y el momento óptimo para su realización deberían basarse en las características y la situación clínica particular de cada paciente, el juicio del médico, el conocimiento de la técnica, la probabilidad de un resultado favorable del procedimiento, los riesgos de la IOT prolongada, las potenciales ventajas de la TRQ, el coste del proceso, la práctica local y la comunicación con las familias.

\section{Bibliografía}

1. Ahmed, N., Yen-Hong, K.: Early versus late tracheostomy in patients with severe traumatic head injury. Surgical infections 2007; 8: 343-348.

2. Arabi, Y., Haddad, S., Shirawi, N., Al Shimemeri, A.: Early tracheostomy in intensive care trauma patients improves resource utilization: a cohort study and literature review. Critical Care 2004; 8: R347-R352.

3. Armstrong, P.A., McCarthy, M.C., Peoples, J.B.: Reduced use of recourses by early tracheostomy in ventilator dependent patients with blunt trauma. Surgery 1998; 124: 763767.

4. Bouderka, M.A., Fakhir, B., Bouaggad, A., Hmamouchi, B., Hamoudi, D., Harti, A.: Early tracheostomy versus prolonged endotracheal intubation in severe head injury. J. Trauma
2004; 57: 251-254

5. Barquist, E.S., Amortegui, J., Hallal, A., et al.: Tracheostomy in ventilator dependent trauma patients: a prospective, randomized intention-to-treat study. J. Trauma 2006; 60: 91-97.

6. Boynton, J.H., Hawkins, K., Eastridge, B.J., O'Keefe, G.E.: Tracheostomy timing and the duration of weaning in patients with acute respiratory failure. Critical Care 2004; 8: R261-R267.

7. Brook, A.D., Sherman, G., Malen, J., Kollef, M.H.: Early versus late tracheostomy in patients who require prolonged mechanical ventilation. Am. J. Crit. Care 2000; 9: 352359.

8. Browd, S.R., MacDonald, J.D.: Percutaneous dilational tracheostomy in neurosurgical patients. Neurocrit. Care 2005; 2: $268-273$.

9. Chia-Lin, H., Kuan-Yu, CH., Chia-Hsuin, CH., JihShuin, J., Chong-Jen, Yu., Pan-Chyr, Y.: Timing of tracheostomy as a determinant of weaning success in critically ill patients: a retrospective study. Critical Care 2005; 9: R46-R52 (DOI 10.1186/cc3018).

10. Díaz-Regañón, G., Miñambres, E., Ruiz, A., GonzálezHerrera, S., Holanda-Peña, M., López-Espadas, F.: Safety and complications of percutaneous tracheostomy in a cohort of 800 mixed ICU patients. Anaesthesia 2008; 63: 1198-1203.

11. Freeman, B.D., Isabella, K., Lin, N., Bucham, T.G.: A meta-analysis of prospective trials comparing percutaneous and surgical tracheostomy in critically ill patients. Chest 2000: $118 ; 1412-1418$.

12. Freeman, B.D., Borecki, I.B., Coopersmith, C.M., Bucham, T.G.: Relationship between tracheostomy timing and duration of mechanical ventilation in critically ill patients. Crit Care Med 2005; 33: 2513-2520.

13. Frutos-Vivar, F., Esteban, A., Apezteguía, C. et al. For the Internacional Mechanical Ventilation Study Group. Outcome of mechanically ventilated patients who require a tracheostomy. Crit Care Med 2005; 33: 290-298.

14. Goettler, C.E., Fugo, J.R., Bard, M.R., et al.: Predicting the need for early tracheostomy: a multifactorial análisis of 992 intubated trauma patients. J. Trauma 2006; 60: 991-996.

15. Griffiths, J., Barber, V.S., Morgan, L., Young, D.: Systematic review and meta-analysis of the studies of the timing of tracheostomy in adult patients undergoing artificial ventilation. BMJ 2005; 330: 1243 (DOI:10.1136/bmj.38467.485671.EO).

16. Groves, D.S., Durbin, C.G. Jr.: Tracheostomy in the critically ill: indications, timing and techniques. Curr Opin Crit Care 2007; 13: 90-97.

17. Gurkin, M.A., Parikshak, M., Kralovich, K.A., Horst H.M., Agarwal, V., Payne, N.: Indicators for tracheostomy in patients with traumatic brain injury. American Surgeon 2002; 68: 324-328.

18. Heffner, J.E.: Medical indications for tracheostomy. Chest 1989; 96: 186-190. 
19. Heffner, J.E.: Timing of tracheostomy in mechanically ventilated patients. Am Rev Respir Dis1993; 147: 768-771.

20. Heffner, J.E.: The role of tracheostomy in weaning. Chest 2001;1 20 Suplement: 477S-481S.

21. Huttner, H.B., Kohrmann, M., Berger, CH., Georgiadis, D., Schwab, S.: Predictive factors for tracheostomy in neurocritical care patients with spontaneous supratentorial hemorraghe. Cerebrovasc Dis 2006; 21: 159-165.

22. Koh, W.Y., Lew, T.W., Chin, N.M., Wong, M.F.: Tracheostomy in a neuro-intensive care setting: indications and timing. Anaesth Intensive Care 1997; 25: 365-368.

23. Kollef, M.H., Ahrens, T.S., Shannon, W.: Clinical predictors and outcomes for patients requiring tracheostomy in intensive care unit. Crit Care Med 1999; 27: 1714-1720.

24. Lee, J.C., Fink, M.P.: Early percutaneous dilatational tracheostomy leads to improved outcomes in critically ill medical patients as compared to delayed tracheostomy. Critical Care 2005; 9; E12 (DOI:10.1186/cc3759).

25. Major, K.M., Hui, T., Wilson, M.T., Gaon, M.D., Shabot, M.M., Margulies,D.R.: Objective indications for early tracheostomy after blunt head trauma. The American Journal of Surgery 2003; 186: 615-619.

26. Moller, M.G., Slaikeu, J.D., Bonelli, P., Davis, A.T., Hoogeboom, J.E., Bonnell, B.W.: Early tracheostomy versus late tracheostomy in the surgical intensive care unit. The American Journal of Surgery 2005; 189: 293-296.

27. Milanchi, S., Magner, D., Wilson, M.T., Mirocha, J., Margulies, D.R.: Percutaneous tracheostomy in neurosurgical patients with intracranial pressure monitoring is safe. $\mathrm{J}$ Trauma 2008; 65: 73-79.

28. Qureshi, A.I., Suárez, J.I., Parekh, P.D., Bhardwaj, A.: Prediction and timing of tracheostomy in patients with infratentorial lesions requiring mechanical ventilatory support. Crit Care Med 2000; 28: 1383-1387.

29. Rello, J., Lorente, C., Díaz, E., et al.: Incidente, etiology, and outcome of nosocomial pneumonia in ICU patients requiring percutaneous tracheotomy for mechanical ventilation. Chest 2003; 124: 2239-2243.
30. Rodríguez, J.L., Steinberg, S.M., Luchetti, F.A., Gibbons, K.J., Taheri, P.A., Flint, L.M.: Early tracheostomy for primary airway management in the surgical critical care setting. Surgery 1990; 108: 655-659.

31. Rumbak, M., Newton, M., Truncale, T., Schwartz, S., Adams, J.W., Hazard, P.B.: A prospective, randomized, study comparing early percutaneous dilational tracheostomy to prolonged translaryngeal intubation (delayed tracheotomy) in critically ill medical patients. Crit Care Med 2004; 32: 16891694.

32. Scales, D.C., Thiruchelvam, D., Kiss, A., Redelmeier, D.A.: The effect of tracheostomy timing during critical illness on long-term survival. Crit Care Med 2008; 36: 2547-2557.

33. Silvester, W., Goldsmith, D., Uchino, S. et al.: Percutaneous versus surgical tracheostomy: a randomized controlled study with long-term follow-up. Crit Care Med 2006; 34: 2145-2152.

34. Schauer, J., Engle, L., Maugher, D., Cherry, R.: Does acuity matter? Optimal timing of tracheostomy stratified by injury severity. J of Trauma 2009; 66: 220-225.

35. Sugerman, H., Luke, W., Pasquale, M. et al.: Multicenter, randomized, prospective trial of early tracheostomy. J of Trauma 1997; 43: 741-747.

36. Zygun, D.A., Zuege, D.J., Boiteau, P.J. et al.: Ventilator-associated pneumonia in severe traumatic brain injury. Neurocrit Care 2006; 5: 108-114.

Gandía-Martínez, F.; Martínez-Gil, I.; Andaluz-Ojeda, D.; Bobillo de Lamo, F.; Parra-Morais, L.; Díez-Gutiérrez, F.: Análisis de la traqueotomía precoz y su impacto sobre la incidencia de neumonía, consumo de recursos y mortalidad en pacientes neurocríticos. Neurocirugía 2010; 21: 211221 .

Correspondencia: Dr. F. Gandía Martínez. Servicio de Medicina Intensiva. Hospital Clínico Universitario. Avenida Ramón y Cajal, 3. 47005 Valladolid.

fgandia@saludcastillayleon.es 